\title{
Diseño de un método para la determinación de las medidas antropométricas para ser usadas en el tallaje de la población peruana
}

\author{
Nicolás Salazar Medina*, Marco Henrich Saavedra*, \\ Patricia Larios Francia*, Martín Reaño Vera* \\ Universidad de Lima \\ George Schofield Bonello* \\ G\&G Consultores Asociados
}

Recibido: 8 de marzo del 2018 / Aprobado: 12 de julio del 2018

doi: 10.26439/ing.ind2018.n036.2447

\begin{abstract}
Resumen: El objetivo de la investigación es establecer las bases para la normalización del tallaje de la población peruana al optimizar recursos, procesos y oferta, alineados a la satisfacción de las necesidades del mercado. Encuestas a empresarios, entrevistas con expertos y mediciones antropométricas revelaron resultados significativos. Basados en estándares internacionales y recomendaciones de expertos, se realizaron 28 mediciones corporales, con lo cual se logró un método para la toma manual de medidas corporales.
\end{abstract}

Palabras clave: tamaño corporal / antropometría - metodología / estatura / cuerpo humano - pesos y medidas / Perú

\section{Designing a method to determine the anthropometric measurements to be used in the clothing sizing of the Peruvian population}

\begin{abstract}
The objective of the research is to establish the bases for the standardization of the Peruvian population clothing sizing by means of optimizing resources, processes and supply, adjusted to the satisfaction of the market needs. Surveys of entrepreneurs, interviews with experts and anthropometric measurements revealed important results. Based on international standards and expert recommendations, 28 body measurements were carried out, achieving a method for taking manual body measurements.
\end{abstract}

Keywords: body size / anthropometry - methodology / height / human body-weights and measures / Peru

* Correos electrónicos: nsalazar@ulima.edu.pe, rlariosf@ulima.edu.pe, mhenrich@ulima.edu.pe, arvera@ulima.edu.pe,grschofield@yahoo.es 


\section{INTRODUCCIÓN}

Los estudios de dimensionamiento a gran escala analizan las dimensiones de una muestra representativa de la población para obtener datos de medidas antropométricas. Sus aplicaciones no se limitan a un sector de la industria; por el contrario, son aplicados a todos los ámbitos de la antropometría: ergonomía, seguridad ocupacional, salud, valoración nutricional, moda y arquitectura (Boueri, 2008; Konz y Johnson, 2008).

Desde hace algún tiempo la industria textil ha considerado estudios de dimensionamiento como una fuente de información sobre los consumidores de acuerdo con sus medidas corporales, su forma y su tamaño. Países como el Reino Unido, Estados Unidos, Tailandia o México han desarrollado estudios de su población en colaboración con diferentes miembros del gobierno, institutos científicos y empresas textiles para elaborar una base de datos nacional.

Ante la falta de una norma actualizada de tallas en el Perú (según indicó la Sociedad Nacional de Industrias), solo existe la NTP-ISO 36352009, que indica el procedimiento para tomar medidas corporales. La presente investigación tiene como objetivo desarrollar una metodología alternativa que permita determinar la antropometría de la población para definir las tallas de las prendas de vestir en el Perú, y confirmar la necesidad de iniciar un proceso automatizado de toma de tallas a nivel nacional. Ello permitiría optimizar la utilización de los recursos, de los procesos y de la diversificación de la oferta para contribuir a una mayor satisfacción de las necesidades de vestido en el mercado y reducir las compras inadecuadas de insumos para su elaboración.

El aporte del presente estudio es la generación de una base de datos de las variables antropométricas de la población que permitirá a los fabricantes desarrollar sus propios modelos de prendas con medidas dentro del rango que se establezca para cada talla; asimismo, se ha desarrollado una metodología estandarizada para la definición de medidas antropométricas, así como la identificación y el análisis de los sistemas de tallas empleados en el mundo.

En opinión de los expertos del sector, una dificultad que afrontan las empresas de confecciones en el Perú es que no cuentan con estudios que les permitan determinar la distribución de tallas que deben ofrecer al mercado, lo cual vienen realizando en la actualidad de acuerdo con los resultados de cada campaña y con sus propias investigaciones, que 
solo tienen un sustento empírico; sin embargo, esto muchas veces les significan quedarse con altos volúmenes de prendas de determinadas tallas y que luego deben tratar de vender como saldos en outlets, con la consiguiente reducción de ingresos y de rentabilidad.

\section{METODOLOGÍA}

El desarrollo de la presente propuesta corresponde a un diseño no experimental transaccional que se realizó entre marzo del 2015 y marzo del 2016. En ese periodo, se recolectaron datos con el propósito de describir las variables y analizar su incidencia (Hernández, Fernández y Baptista, 2010). La metodología del estudio incluyó la utilización de las siguientes fuentes primarias: encuestas a empresarios, entrevistas a expertos del sector textil de confecciones y mediciones antropométricas. Del análisis y la obtención de datos preliminares y finales de la encuesta se alcanzaron resultados cualitativos y cuantitativos. Se utilizaron sistemas de análisis de datos y se realizó un estudio antropométrico de las 28 dimensiones que involucró la determinación de las medidas críticas mínimas para tipificar la antropometría de la población.

En la fase de campo de la investigación se aplicó una metodología manual para el acopio de datos en 2D. Los resultados circunscritos a una muestra limitada de individuos localizados en Lima han sido utilizados para obtener datos sobre sus características antropométricas. Nuevos estudios han permitido introducir la tecnología del escáner 3D (volumétrica) de alta producción en escala, la cual ofrece una mayor confiabilidad de los resultados. Esta metodología permite incorporar los criterios morfológicos y las proporciones corpóreas que tipifican la riqueza étnica de la población peruana.

Para la metodología de la medición antropométrica, se tomaron como referencia los estándares para medidas corporales de la norma internacional ASTM D5219 (American Society for Testing Materials), de las normas andinas NA 0026, NA 0027 y NA 0028 y de métodos de medición de expertos en moldaje y construcción de prendas del sector textil de confecciones en el Perú.

Como resultado de la comparación de las normas técnicas internacionales, se han estimado 28 medidas antropométricas, las cuales se han considerado como las más utilizadas para la confección de prendas textiles. Asimismo, se incluyó el registro del peso de los individuos (ver tabla 1). 
Tabla 1

Matriz de principales medidas antropométricas

\begin{tabular}{|c|c|c|c|c|c|c|}
\hline \multirow[b]{2}{*}{1} & \multicolumn{2}{|l|}{ Medida } & \multirow{2}{*}{$\begin{array}{c}\text { ASTM } 5219 \\
\mathrm{X}\end{array}$} & \multirow{2}{*}{$\begin{array}{r}\text { NA } 26 \\
x\end{array}$} & \multirow{2}{*}{$\begin{array}{r}\text { NA } 27 \\
x\end{array}$} & \multirow{2}{*}{$\begin{array}{r}\text { NA } 28 \\
X\end{array}$} \\
\hline & Subject Height & Altura del sujeto & & & & \\
\hline 2 & Head Circumference & $\begin{array}{l}\text { Circunferencia de } \\
\text { la cabeza }\end{array}$ & $x$ & & $x$ & \\
\hline 3 & Collar Circumference & $\begin{array}{l}\text { Circunferencia del } \\
\text { cuello base }\end{array}$ & $\mathrm{X}$ & $x$ & $x$ & $x$ \\
\hline 4 & $\begin{array}{l}\text { Back Shoulder Width } \\
\text { Horizontal }\end{array}$ & $\begin{array}{l}\text { Ancho del hombro } \\
\text { posterior horizontal }\end{array}$ & $\mathrm{x}$ & $x$ & $x$ & $\mathrm{X}$ \\
\hline 5 & Shoulder Slope & Caída del hombro & $x$ & $\mathrm{x}$ & $x$ & $x$ \\
\hline 6 & Shoulder Length & Ancho del hombro & $\mathrm{x}$ & $x$ & $x$ & $x$ \\
\hline 7 & $\begin{array}{l}\text { Across Axilla Chest } \\
\text { Front Length }\end{array}$ & $\begin{array}{l}\text { Ancho de pecho } \\
\text { frontal }\end{array}$ & $x$ & $x$ & $x$ & $x$ \\
\hline 8 & $\begin{array}{l}\text { Across Axilla Chest } \\
\text { Back Length }\end{array}$ & Ancho de espalda & $x$ & $x$ & $x$ & $x$ \\
\hline 9 & $\begin{array}{l}\text { Arm Hole } \\
\text { Circumference }\end{array}$ & $\begin{array}{l}\text { Circunferencia de } \\
\text { la sisa }\end{array}$ & $x$ & $\mathrm{X}$ & $x$ & $x$ \\
\hline 10 & Arm Length & Longitud del brazo & $x$ & $x$ & $x$ & $x$ \\
\hline 11 & Bust To Bust Length & $\begin{array}{l}\text { Ancho de busto a } \\
\text { busto }\end{array}$ & $\mathrm{x}$ & $x$ & & $x$ \\
\hline 12 & $\begin{array}{l}\text { Vertical Trunk } \\
\text { Circumference }\end{array}$ & $\begin{array}{l}\text { Circunferencia } \\
\text { vertical del tronco }\end{array}$ & $\mathrm{x}$ & $x$ & $x$ & $x$ \\
\hline 13 & $\begin{array}{l}\text { Chest / Bust } \\
\text { Circumference }\end{array}$ & $\begin{array}{l}\text { Circunferencia del } \\
\text { busto / pecho }\end{array}$ & $x$ & $x$ & $x$ & $x$ \\
\hline 14 & $\begin{array}{l}\text { OverArm } \\
\text { Circumference }\end{array}$ & $\begin{array}{l}\text { Circunferencia } \\
\text { sobre el brazo }\end{array}$ & $x$ & $x$ & $x$ & $x$ \\
\hline 15 & Waist Circumference & $\begin{array}{l}\text { Circunferencia de } \\
\text { la cintura }\end{array}$ & $x$ & $x$ & $x$ & $x$ \\
\hline 16 & Waist Height & Altura de la cintura & $\mathrm{x}$ & $x$ & $x$ & $x$ \\
\hline
\end{tabular}

(continúa) 
(continuación)

\begin{tabular}{|c|c|c|c|c|c|c|}
\hline & Medida & & ASTM 5219 & NA 26 & NA 27 & NA 28 \\
\hline 17 & High Hip & $\begin{array}{l}\text { Circunferencia de } \\
\text { la cadera alta }\end{array}$ & $x$ & $x$ & & $x$ \\
\hline 18 & Wrist Circumference & $\begin{array}{l}\text { Circunferencia de } \\
\text { la muñeca }\end{array}$ & $x$ & $x$ & $x$ & $x$ \\
\hline 19 & Hip Circumference & $\begin{array}{l}\text { Circunferencia de } \\
\text { la cadera }\end{array}$ & $x$ & $X$ & $x$ & $x$ \\
\hline 20 & Crotch Height & $\begin{array}{l}\text { Altura de la } \\
\text { entrepierna }\end{array}$ & $\mathrm{X}$ & $X$ & $x$ & $\mathrm{X}$ \\
\hline 21 & Crotch Length Full & $\begin{array}{l}\text { Longitud de } \\
\text { la entrepierna } \\
\text { completa }\end{array}$ & $\mathrm{X}$ & $X$ & $x$ & $\mathrm{X}$ \\
\hline 22 & Thigh Circumference & $\begin{array}{l}\text { Circunferencia del } \\
\text { muslo }\end{array}$ & $x$ & $X$ & $\mathrm{X}$ & $\mathrm{x}$ \\
\hline 23 & $\begin{array}{l}\text { MidThigh } \\
\text { Circumference }\end{array}$ & $\begin{array}{l}\text { Circunferencia del } \\
\text { muslo medio }\end{array}$ & $x$ & $x$ & $x$ & \\
\hline 24 & Knee Circumference & $\begin{array}{l}\text { Circunferencia de } \\
\text { la rodilla }\end{array}$ & $x$ & $x$ & $x$ & $\mathrm{X}$ \\
\hline 25 & Calf Circumference & $\begin{array}{l}\text { Circunferencia de } \\
\text { la pantorrilla }\end{array}$ & $x$ & $x$ & $x$ & $x$ \\
\hline 26 & Ankle Circumference & $\begin{array}{l}\text { Circunferencia del } \\
\text { tobillo }\end{array}$ & $x$ & $\mathrm{X}$ & $x$ & $\mathrm{X}$ \\
\hline 27 & Foot Width & Ancho del pie & $x$ & & $\mathrm{x}$ & \\
\hline 28 & Foot Length & Longitud del pie & $\mathrm{X}$ & & $X$ & \\
\hline
\end{tabular}

Elaboración propia

Para la muestra inicial de las variables antropométricas para la industria textil, se midieron a 100 personas: 56 hombres y 44 mujeres. El estudio definió este tamaño de muestra preliminar a un $95 \%$ del nivel de confianza 5,8 \% de error. Se propuso trabajar en el estudio final con un margen de error de $2 \%$, y llegar así a un tamaño de muestra de 471 para varones y 278 para mujeres. 
Se ha verificado que, si bien la metodología de medición antropométrica manual es muy útil para poder contar con esta información para la industria, al mismo tiempo también puede significar la posibilidad de incurrir en errores de toma de datos; además, se ha evidenciado que el tiempo estándar de cada medición haría innecesariamente extenso y costoso un estudio a escala nacional.

De acuerdo con criterios internacionales, se realizó una investigación en la industria peruana, y se determinaron las medidas antropométricas de la población necesarias para la elaboración de prendas de vestir y se tomaron como referencia las normas andinas elaboradas por el Comité Andino de Normalización y de ASTM International, así como la opinión experta de industriales de la confección en el Perú. La tabla 1 compara la inclusión de las medidas antropométricas más relevantes que son consideradas en las principales normas internacionales.

\section{ANÁLISIS ANTROPOMÉTRICO}

Para definir los equipos y las herramientas para la medición, la investigación desarrolló un método para la toma de medidas corporales de manera manual. Para ello, se utilizaron equipos y herramientas que fueron adquiridos especialmente para llevar a cabo el estudio, razón por la cual no se tuvieron que calibrar. Dichos equipos y herramientas fueron: una báscula digital de columna profesional, un antropómetro (Large Bone Caliper Lafayette), un estadiómetro de acero flexible, una cinta métrica flexible y una herramienta de nivel de burbuja.

El estudio consideró un tamaño de muestra preliminar de 56 hombres y 44 mujeres para las mediciones. Para determinar que la colección de los datos en las diferentes medidas (tanto para hombres como para mujeres) correspondiera a una distribución normal, se utilizó la prueba de bondad de ajuste de Kolmogoroff-Smirnoff, la cual dio como resultado que las medidas se ajustaran a una distribución normal. Todos los datos que se presentan como resultado preliminar representan a la población de Lima Metropolitana. Los datos del censo del 2007 del Instituto Nacional de Estadística e Informática (INEI) permitirán determinar los rangos de edades más adecuados para el desarrollo de las mediciones. 


\section{MEDIDAS CORPORALES DE LA INVESTIGACIÓN ANTROPOMÉTRICA}

Para la presente investigación se ha considerado el estudio de 28 medidas corporales. Algunas de ellas detallan la forma de medir a continuación:

- Peso y altura del sujeto

Con la ayuda de la báscula, se realiza el pesaje exacto de manera digital; asimismo, se calcula el índice de masa corporal (IMC) y, con el estadiómetro, se mide la distancia vertical desde la coronilla de la cabeza hasta la base de los pies, tomada con el sujeto de pie $\mathrm{y}$ sin zapatos.

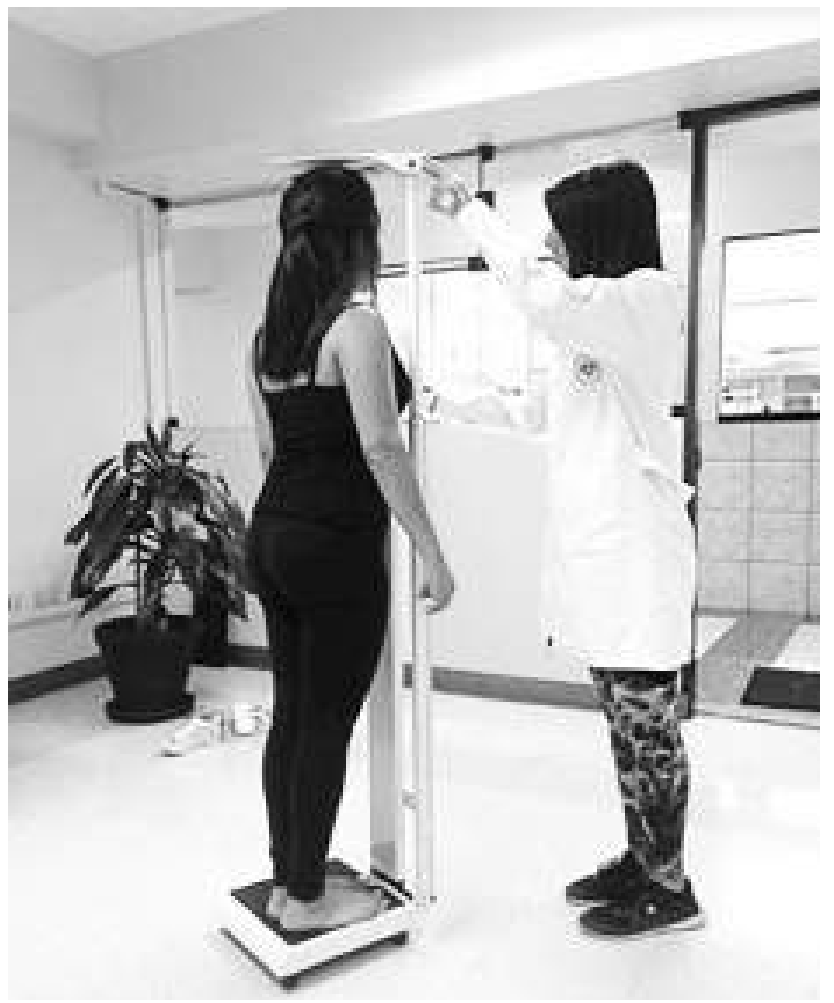

Figura 1. Peso y altura del sujeto*

* Las fotografías de este artículo han sido tomadas por Patricia Larios. 
- Circunferencia de la cabeza

Con el estadiómetro, se mide el perímetro de la cabeza sobre las orejas.

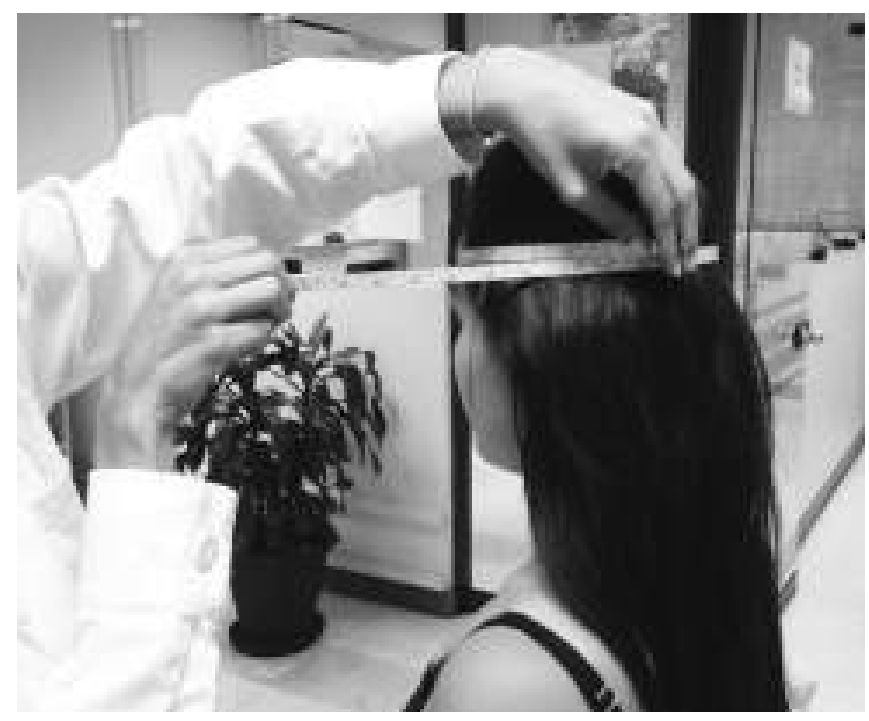

Figura 2. Circunferencia de la cabeza

- Circunferencia de la base del cuello

Con el estadiómetro, se mide la circunferencia del cuello, tomada sobre la cervical y en la parte superior de la clavícula.

- Circunferencia del busto/pecho

Con la cinta métrica flexible, se mide la circunferencia horizontal alrededor del torso, tomada bajo los brazos y en la parte más completa del ápice del pecho (hombres) y busto (mujeres), incluida la parte inferior de los omóplatos.

- Circunferencia de la cadera alta

Con la cinta métrica flexible, se mide la máxima circunferencia horizontal alrededor del torso, tomada por debajo del nivel de la cintura e incluida la extensión abdominal.

- Circunferencia de la cadera alta

Con la cinta métrica flexible, se mide la máxima circunferencia horizontal alrededor del torso, tomada por debajo del nivel de la cintura e incluida la extensión abdominal. 
- Ancho del hombro posterior horizontal

Con en el antropómetro Large Bone Caliper, se mide la distancia horizontal a través de la espalda de la parte superior del hombro en la intersección de la clavícula con cabeza del hueso húmero.

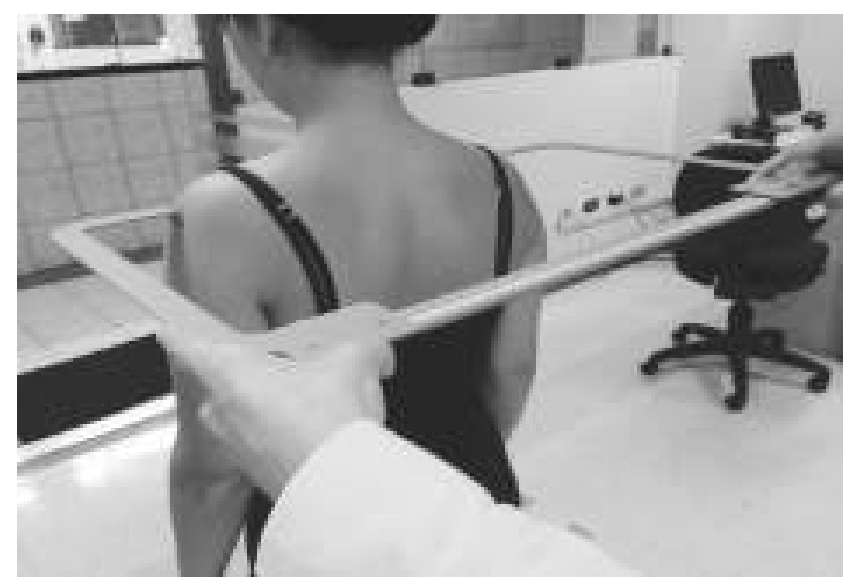

Figura 3. Ancho del hombro posterior horizontal

- Ancho de espalda

La distancia horizontal es medida con la ayuda del antropómetro a través de la espalda de axila a axila.

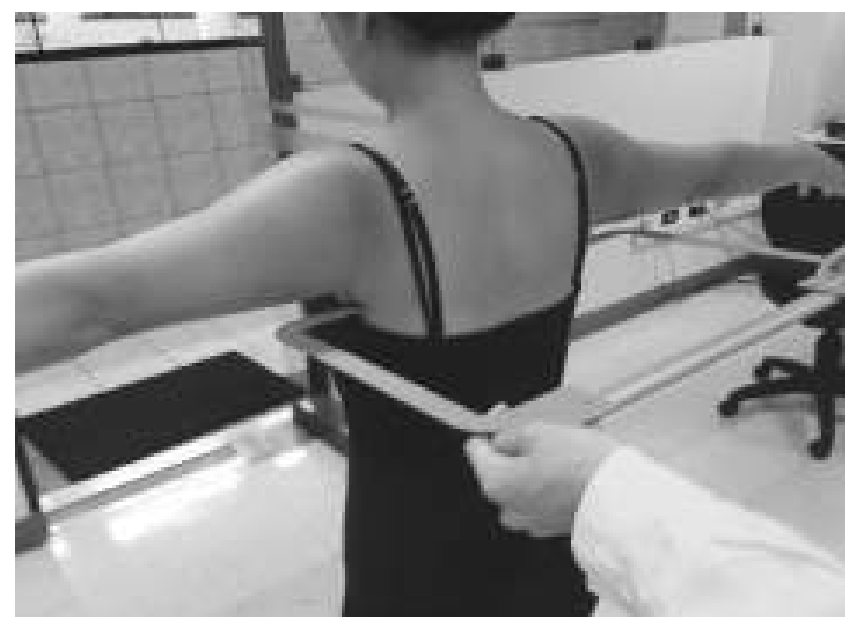

Figura 4. Ancho de espalda 
- Circunferencia del tobillo

Con la cinta métrica flexible, se mide la máxima circunferencia horizontal del tobillo, tomada sobre la mayor prominencia de los huesos del tobillo.

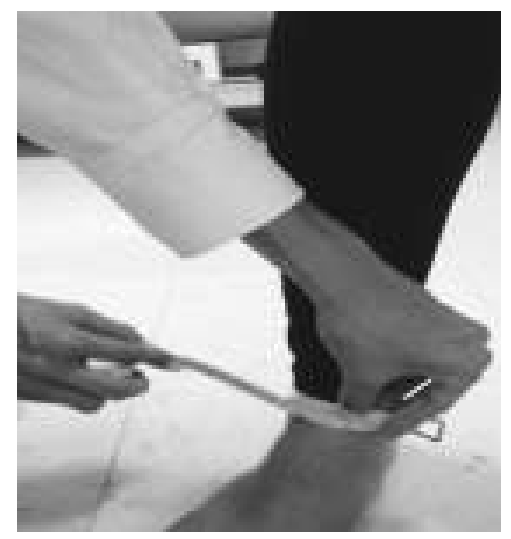

Figura 5. Circunferencia del tobillo

- Ancho del pie

La distancia recta es tomada con la ayuda de un antropómetro, de un lado del pie al otro en la parte más ancha, y tomada con el sujeto de pie y sin zapatos.

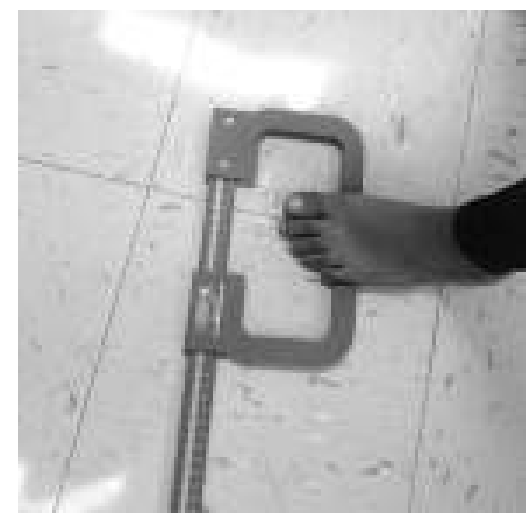

Figura 6. Ancho del pie 


\section{RESULTADOS}

Se realizó una encuesta a empresarios (muestra conformada por 30 gerentes, supervisores y especialistas del sector textil de confecciones) y se obtuvo como resultado que el $80 \%$ consideraba necesario contar con un sistema de tallas estandarizadas. Esto es particularmente importante, ya que el $83 \%$ de estos, a su vez, identificaron la existencia de diferencias entre las tallas que producen para el mercado externo respecto a las que producen para el mercado nacional.

De acuerdo con las encuestas, entrevistas a expertos y estándares internacionales, se determinaron las medidas antropométricas de la población necesarias para la elaboración de prendas de vestir, y se tomaron como referencia las normas andinas elaboradas por el Comité Andino de Normalización y de ASTM International, así como la opinión experta de industriales de la confección en el Perú.

Cabe destacar que, como resultado de la encuesta, se determinó que el $77 \%$ de confeccionistas de prendas en el Perú utiliza algún estándar de tallas, mientras que el $23 \%$ de estos confeccionistas utiliza un estándar propio.

El estudio permite un primer acercamiento a una distribución proporcional para estimar lotes de producción a las empresas del rubro y reducir el margen de error de la cantidad de prendas que deberían producir para cada campaña. En la tabla 2, se aprecia la distribución de los 100 individuos que conformaron la muestra:

Tabla 2

Total de sujetos medidos

\begin{tabular}{lccccccc}
\hline \multicolumn{7}{c}{ Rango de edades } \\
\hline Género & $\mathbf{1 8 - 2 5}$ & $\mathbf{2 6 - 3 5}$ & $\mathbf{3 6 - 4 5}$ & $\mathbf{4 6 - 5 5}$ & $\mathbf{5 6 - 6 4}$ & $\mathbf{6 5 +}$ & Total \\
Masculino & 10 & 13 & 10 & 12 & 7 & 4 & 56 \\
Femenino & 10 & 12 & 8 & 6 & 4 & 4 & 44 \\
\hline
\end{tabular}

Elaboración propia

A partir de las medidas antropométricas promedio obtenidas, el empresario peruano contará con datos que le permitirán revisar los sistemas de tallas que vienen utilizando actualmente.

Como resultado de las mediciones antropométricas realizadas a la muestra, se obtuvo como resultado: 
Tabla 3

Medidas antropométricas promedio

\begin{tabular}{|c|c|c|c|c|}
\hline \multirow[b]{3}{*}{ Medida (metros) } & \multirow{2}{*}{\multicolumn{2}{|c|}{ Mujeres }} & & \\
\hline & & & \multicolumn{2}{|c|}{ Varones } \\
\hline & Media & $\begin{array}{l}\text { Desviación } \\
\text { estándar }\end{array}$ & Media & $\begin{array}{c}\text { Desviación } \\
\text { estándar }\end{array}$ \\
\hline Altura & 155.325 & 5.911 & 166.556 & 6.424 \\
\hline Circunferencia de la cabeza & 55.220 & 1.907 & 56.104 & 1.954 \\
\hline Circunferencia del cuello & 32.698 & 2.778 & 39.467 & 3.121 \\
\hline Ancho del hombro posterior horizontal & 36.590 & 1.844 & 42.517 & 2.158 \\
\hline Inclinación del hombro & 18.675 & 3.174 & 17.722 & 3.921 \\
\hline Longitud del hombro & 13.613 & 1.487 & 16.222 & 1.519 \\
\hline Ancho del pecho frontal & 50.250 & 4.970 & 52.769 & 3.911 \\
\hline Circunferencia del busto/ pecho & 93.100 & 8.745 & 100.585 & 8.306 \\
\hline Ancho de espalda & 29.225 & 2.437 & 33.185 & 2.634 \\
\hline Circunferencia de la sisa & 35.050 & 4.035 & 39.778 & 4.652 \\
\hline Longitud del brazo & 55.488 & 3.056 & 58.713 & 3.049 \\
\hline Longitud de busto a busto & 18.313 & 2.314 & - & - \\
\hline Circunferencia vertical del tronco & 150.765 & 6.116 & 170.113 & 7.043 \\
\hline Longitud de la entrepierna completa & 72.125 & 4.275 & 86.426 & 6.596 \\
\hline Circunferencia sobre el brazo & 26.273 & 2.463 & 29.396 & 3.155 \\
\hline Circunferencia de la cintura & 79.380 & 10.874 & 93.333 & 10.773 \\
\hline Altura de la cintura & 99.938 & 5.662 & 106.620 & 5.999 \\
\hline Circunferencia de la cadera alta & 93.638 & 9.448 & 97.583 & 8.063 \\
\hline Circunferencia de la cadera & 96.333 & 6.532 & 100.570 & 6.881 \\
\hline Circunferencia de la muñeca & 15.350 & 1.222 & 17.154 & 1.029 \\
\hline Altura de la entrepierna & 67.675 & 5.298 & 71.759 & 5.757 \\
\hline Circunferencia del muslo & 53.168 & 3.366 & 53.381 & 4.433 \\
\hline Circunferencia del muslo medio & 47.080 & 3.396 & 47.176 & 4.616 \\
\hline Circunferencia de la rodilla & 36.265 & 2.300 & 37.639 & 3.388 \\
\hline Circunferencia de la pantorrilla & 33.613 & 2.327 & 35.820 & 3.096 \\
\hline Circunferencia del tobillo & 23.453 & 1.974 & 25.156 & 2.036 \\
\hline Ancho del pie & 8.728 & 0.580 & 9.985 & 0.848 \\
\hline Longitud del pie & 22.800 & 1.410 & 25.272 & 1.189 \\
\hline Peso (kilos) & 58.605 & 8.865 & 75.533 & 12.164 \\
\hline
\end{tabular}

Elaboración propia 
El análisis cuantitativo de las medidas antropométricas de la muestra concluyó que el tamaño de muestra para un nivel de confianza del $95 \%$ y un margen de error del $5 \%$ es de 77 individuos. El presente estudio evaluó 100 sujetos. Para ser más exigentes, se propone trabajar con un margen de error del $2 \%$, y llegar así a un tamaño de muestra de 471 para varones y 278 para mujeres para el estudio final; sin embargo, experiencias de otros países señalan que la toma de medidas se debe realizar a un espectro muestral lo más amplio posible.

Dada la cantidad de sujetos que se requerirán en el estudio final, un estudio antropométrico confiable a escala nacional requiere de escáneres corporales 3D que permitan obtener datos antropométricos de todo el cuerpo humano de forma rápida y precisa y sin invadir la privacidad de las personas, ya que estos equipos están diseñados para tomar mediciones a partir de aproximaciones en las que no se tiene contacto directo con el cuerpo. A ello se suma la necesidad de trabajar con sistemas que posibiliten ser transportados a diferentes lugares a lo largo del país y que no requieran de calificación técnica para instalarlos y desinstalarlos.

\section{CONCLUSIONES}

Se encontró que en la industria de la confección peruana no hay un sistema de tallas estándar, ya que no se ha encontrado evidencia de existencia de valores antropométricos de la población peruana.

En opinión de los expertos del sector, una dificultad que afrontan muchas empresas confeccionistas en el Perú es que no se cuenta con estudios que les permitan determinar la distribución de tallas que deben ofrecer al mercado, lo que trae como consecuencia quedarse con altos volúmenes de prendas y que luego deben tratar de vender como saldos en "outlets", con la consiguiente reducción de ingresos y rentabilidad.

Este estudio permite un primer acercamiento a una distribución proporcional que permita a las empresas poder estimar con un menor margen de error la cantidad de prendas que deberían producir de acuerdo con el patrón de tallas que estas manejen en sus propios negocios.

Se definió una metodología para establecer una tabla antropométrica de la población peruana que se utilice para definir los sistemas de tallas en prendas de vestir.

A partir de las medidas antropométricas promedio obtenidas en el presente estudio, el empresario peruano contará con una herramienta 
basada en datos preliminares para revisar sus sistemas de tallas utilizados actualmente, lo que determinará en la optimización de sus recursos.

La toma manual tuvo un tiempo promedio de 13 minutos por cada sujeto, con el apoyo de tres técnicos debidamente entrenados. Se evidenció que el uso de una metodología de toma de medidas manual causó incomodidad en varias de las personas que aceptaron colaborar con el estudio por razones de pudor o incomodidad en la ejecución de la toma de medidas.

\section{RECOMENDACIONES}

Se recomienda un estudio de la población con mayor alcance territorial que determine resultados de las medidas antropométricas del total de la población peruana, tomando en cuenta las diferentes etnias a lo largo de las regiones del Perú.

Un estudio a nivel nacional debería contar con la participación de la academia, el gobierno, los gremios y la industria.

Los resultados de la investigación pueden ser utilizados como herramienta metodológica y como base de datos para futuros estudios en sectores como salud, estadística, industria de alimentos, manufacturera de muebles y otros.

La investigación obtuvo datos que pueden ser utilizados por la industria del calzado, como el ancho y la longitud del pie. En el estudio también se obtuvieron datos a ser utilizados en la confección de accesorios, prendas complementarias e industria de la joyería (circunferencia de cabeza, circunferencia de cuello y circunferencia de muñeca).

Del análisis estocástico de los resultados, se podrán establecer los parámetros representativos y sus rangos que al escalarlos tipifiquen las tallas de las categorías de prendas que conforman el vestuario básico, según género y edad. Se podrán crear los modelos físicos o virtuales (maniquíes) normalizados que sirvan para el modelado, patronado y escalado de moldes y desarrollos de moda.

Se recomienda, a partir de los resultados del presente estudio, proponer la elaboración de una norma técnica de formas de medición para ser utilizada en la industria de la confección de prendas de vestir y accesorios en tejido de punto, plano, cuero y ganchillo. 


\section{REFERENCIAS}

Ashdown, S. (ed.). (2007). Sizing in clothing. Cambridge: Woodhead Publishing.

ASTM International. (2013). ASTM standards for body measurements. Baltimore: MD.

Berríos, A. I. (2009). La Antropometría en la Moda. Criterios de identificación del consumidor. En O. Echevarría (dir.), Actas de Diseño. Facultad de Diseño y Comunicación. Buenos Aires: Universidad de Palermo.

Boueri Filho, J. (2008) Antropometria aplicada a aquitetura, urbanismo $e$ desenho industrial. São Paulo: Estação das Letras e Cores Editora

Castano, I. (29 de marzo de 2012). Study Focuses on Mexican Sizing. Cámara Nacional de la Industria del Vestido. Recuperado de http://www.canaive.org.mx /detalleNoticias.php?id_nota=878

Centro para el control y la prevención de enfermedades (2012). Ergonomía (desórdenes musculo esqueléticos). Recuperado de http://www.cdc.gov/spanish/niosh/topics/ergonomia.html

Charoensiriwath, S. y Srichaikul, P. (2009). Size Thailand: Constructing Thailand's National Anthropometrics Database using 3D Body Scanning Technology. Centro Nacional de Electrónica y Tecnología Informática de Tailandia. Recuperado de http://www. pnclink.org/pnc2009/chinese/PresentationMaterial/Oct08/08ConfHall-UnfoldingeCulture/08-UnfoldingeCulture-piyawut.pdf

Croem (s. f.). Prevención de Riesgos Ergonómicos. Murcia: Instituto de Seguridad y Salud laboral de la Región de Murcia. Recuperado de http://www.croem.es/prevergo/formativo/1.pdf

Expo Textil Perú 2015 (s. f.) Perú en Cifras. Recuperado de http:// expotextilperu.com/

Faust, M. E. y Carrier, S. (ed.). (2014). Designing apparel for consumers: The impact of body shape and size. Cambridge: Woodhead Publishing Limited.

Fernández, C., Hernández, R. y Baptista, P. (2010). Metodología de la investigación (5. ${ }^{\mathrm{a}}$ ed.). México D.F.: McGraw-Hill. 
Instituto Nacional de Calidad [Inacal]. (2009). Normas Técnicas Peruanas de Textiles. Recuperado de https://www.inacal.gob.pe/ repositorioaps/data/1/1/1/jer/prensa/files/TEXTILES.pdf

Instituto Nacional de Estadística e Informática [INEI] (2015). Comportamiento de la Economía Peruana en el Primer Trimestre de 2015. Recuperado de http://www.inei.gob.pe/ media/MenuRecursivo/boletines/informe-tecnico-n02_pbitrimestral_2015i.pdf

Instituto Nacional de Salud [INS]. (1998). Módulo medidas antropométricas, registro y estandarización. Lima: INS.

INT prepara projeto de pesquisa antropométrica 3D da populaçao. (4 de agosto del 2013). Recuperado de http://www.int.gov.br/component/ docman/doc_view/47404ago2013jornalbrasilantropometria

Just-Style: Mexico National Sizing Survey to begin next year (27 de octubre del 2003). Recuperado de http://www.just-style.com/ news/national-sizing-survey-to-begin-next-year_id67804.aspx

Konz, S. y Johnson, S. (2008). Work Design: occupational ergonomics. Arizona: Holcomb Hathaway Publishers.

Kuttner, D. (2004). What's on with WEAR. WEAR: The World Engineering Anthropometry Resource. Recuperado de http:// www.bodysizeshape.com/newsletterWEAR

Lafayette Instrument. (29 de febrero del 2016). Recuperado de http:// www.lafayettelifesciences.com/product_detail.asp?itemid=2200

London, U. O. (s.f.). Size UK -Results from the UK National Sizing Survey. Recuperado de http://www.arts.ac.uk/

Norma Andina 0026:2007, Tabla Estándar de Medidas Corporales relacionadas con Mujeres con figura tipo talla extra, tallas $14 \mathrm{w}$ 32 w. (25 de julio del 2017). Comunidad Andina.

Norma Andina 0027:2007, Tablas Estándar de Medidas Anatómicas para Hombres de talla 34 a 60 regular. (25 de julio del 2007). Comunidad Andina.

Norma Andina 0028:2007, Tablas Estándar de Medidas Corporales Juveniles, tallas 0 a 19. (25 de julio del 2017). Comunidad Andina.

Parra, N. L. y Rico, A. (2007). Estudio antropométrico de preadolescentes en la ciudad de Ambato (tesis doctoral, Pontificia Universidad Católica del Ecuador, Ecuador). 
Pheasant, S. y Haslegrave, C. M. (2005). Bodyspace: Anthropometry, ergonomics and the design of work. Florida: CRC Press.

Quaresma, M., de Moraes, A. y Cardoso, V. M. B. (2000). Some Causes of Errors Using Anthropometric Data when Designing Products and Workstations. Proceedings of the Human Factors and Ergonomics Society Annual Meeting, 44(33), 323-326.

Roach, K. (31 de julio del 2002). Sizing Survey Benefits Many Manufacturers. Recuperado de http://scm.ncsu.edu/scm-articles/ article/sizing-survey-benefits-many-manufacturers

Roebuck, J. (1995). Anthropometric methods: Designing to fit the human body. California: Human Factors \& Ergonomics Society

Salazar, N., Henrich, M., Larios, R., Reaño, M. y Schofield, G. (2016). Análisis antropométrico para la normalización del tallaje de la población peruana. Lima: IDIC. Universidad de Lima.

Sizemic (s.f.). SizeUK - The National Sizing Survey. Recuperado de http://www.arts.ac.uk/research/research-projects/completedprojects/sizeuk-results-from-the-uk-national-sizing-survey/

Triveño, G. (25 de octubre del 2013). Sector textil genera S/.6.600 mlls. de valor agregado. El Comercio. Recuperado de http:// elcomercio.pe/economia/peru/gladys-triveno-sector-textilgenera6600-mlls-valor-agregado-noticia-1649730?ref=flujo_ tags_ 6656\&ft=nota_19\&e=imagen.

Woolworths invites public to be part of its national sizing survey. (14 de febrero de 2014). Recuperado de http://www.marketingupdate. co.za/?IDStory $=59494$

Zamberlan, M.C. (2013). Cientistas usam scanner tridimensional para descobrir e disponibilizar as verdadeiras medidas da população brasileira. Recuperado de http://www.canalciencia. ibict.br/pesquisa/0146-Medidas-antropometricas-da-populacaobrasileira.html

Zernike, K. (1 de marzo del 2004). Sizing Up America: Signs of Expansion From Head to Toe. New York Times. Recuperado de http://www.nytimes.com/2004/03/01/us/sizing-up-america-signsof-expansion-from-head-to-toe.html?pagewanted=1 
